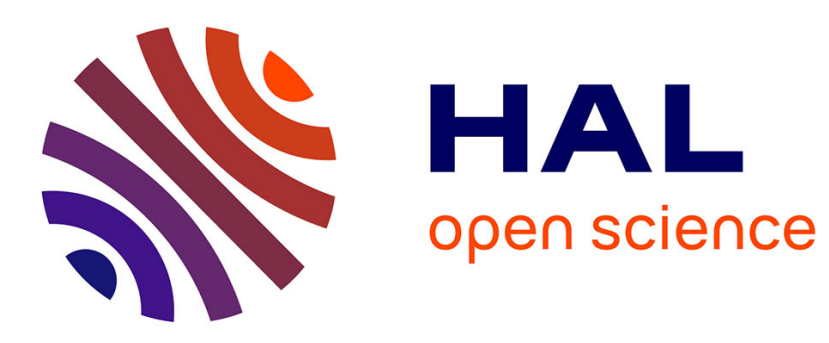

\title{
Elastic relaxations and interactions on metallic vicinal surfaces: testing the dipole model \\ Geoffroy Prévot, Bernard Croset
}

\section{To cite this version:}

Geoffroy Prévot, Bernard Croset. Elastic relaxations and interactions on metallic vicinal surfaces: testing the dipole model. Physical Review B: Condensed Matter and Materials Physics (1998-2015), 2006, 74 (23), pp.235410. 10.1103/PhysRevB.74.235410 . hal-00510745

\section{HAL Id: hal-00510745 \\ https://hal.science/hal-00510745}

Submitted on 20 Aug 2010

HAL is a multi-disciplinary open access archive for the deposit and dissemination of scientific research documents, whether they are published or not. The documents may come from teaching and research institutions in France or abroad, or from public or private research centers.
L'archive ouverte pluridisciplinaire HAL, est destinée au dépôt et à la diffusion de documents scientifiques de niveau recherche, publiés ou non, émanant des établissements d'enseignement et de recherche français ou étrangers, des laboratoires publics ou privés. 


\title{
Elastic relaxations and interactions on metallic vicinal surfaces: testing the dipole model
}

\author{
G. Prévot and B. Croset
}

\begin{abstract}
Institut des NanoSciences de Paris, UMR CNRS 7588, Université Pierre et Marie Curie-Paris 6, Université Denis Diderot-Paris 7, 140, rue de Lourmel, 75015 Paris, France
\end{abstract}

\begin{abstract}
We have studied, by quenched molecular dynamics (QMD) using a second-moment approximation (SMA) potential, the atomic relaxations and step interaction energies on $\mathrm{Ni}$, $\mathrm{Cu}, \mathrm{Pt}$ and $\mathrm{Au}$ vicinal surfaces for which steps run along the $[1 \overline{1} 0]$ direction. The results have been compared to anisotropic linear elasticity calculations (ALE). We show that steps are well described with a model of lines of force dipoles buried under the surface. The elastic interaction energies between steps obtained by SMA and ALE are in good agreement. This demonstrates that the elastic step interaction energy can be determined from the measurement of the atomic relaxations.
\end{abstract}

\section{Introduction}

Vicinal surfaces are obtained by cutting a crystal close to a dense plane. As a consequence, they consist of terraces separated by steps. Steps often display particular properties. For example, they can act as nucleation centres for the growth of metallic nanowires ${ }^{1}$. In that case, the regularity of the wire organization obtained is given by the 
regularity of the array of steps of the bare surface. At finite temperature, steps fluctuate and their correlations are governed by the step-step interactions and the kink creation energy. Whereas the kink creation energy is a very local energetic parameter, step interactions have a long range component. Different contributions to the step interactions can be distinguished. Steps entropically repell through the condition that two steps cannot cross each other ${ }^{2}$. When the steps are close together, the number of allowed configurations are reduced, and this reduction of entropy is equivalent to a step repulsion. Steps interact also electronically through the modification of the density of states $^{3,4}$, electrostatic ally due to the presence of electrostatic dipoles at the steps ${ }^{5,6}$, and thermally through the modification of their vibrational free energy ${ }^{7}$. They also interact elastically through the long range relaxation fields generated by local atomic relaxations at the steps $^{8}$. At low temperature, entropic and phononic contributions are negligible and only electronic, electrostatic and elastic contributions have to be considered. Electronic interactions display an oscillatory behaviour with an exponential decay $^{3}$ whereas electrostatic and elastic interactions are both inversely proportional to the square of the interstep distance ${ }^{8}$. Thus, for small miscut vicinals, corresponding to large interstep distances, steps interact only electrostatically or elastically.

In the past, step interactions have been determined through the measurement by Scanning Tunneling Microscopy (STM) of step fluctuations ${ }^{9}$. It has been recently shown that the step elastic interactions could be deduced from Grazing Incidence X-ray Diffraction (GIXD) measurements of the relaxation fields ${ }^{10,11}$. In this approach, steps are described as elastic dipoles whose value is adjusted in order to fit the experimental measurements. Elastic calculations can then be used to determine the interaction energy between the dipole lines ${ }^{12}$. For $\mathrm{Pt}$ and $\mathrm{Cu}$ vicinals, it was shown that the elastic interaction energy was much higher than the electrostatic contribution ${ }^{10,11}$. This is certainly a general behavior for metallic surfaces, whereas higher dipolar interactions could be probably found on ionocovalent surfaces. 
In this paper, we address the question of whether one can really obtain the elastic step interaction energy by fitting the atomic relaxations with a model of elastic dipoles. In order to answer this question, we have performed Quenched Molecular Dynamics calculations of the atomic relaxations and step interactions on various vicinal surfaces of four transition metals $(\mathrm{Ni}, \mathrm{Cu}, \mathrm{Pt}, \mathrm{Au})$, using a semi-empirical atomistic potential, and compared the values found to the result of linear elasticity calculations using a model of elastic dipoles.

The first part of the paper is devoted to the molecular dynamics computational details. The results obtained for the relaxations and interaction energies are given in the two following parts. A comparison is then made with anisotropic linear elasticity calculations.

\section{Computational details}

\section{A. Geometrical details}

We have studied vicinal surfaces of fcc crystals with dense steps, i.e. steps running along the $[1 \overline{1} 0]$ direction. Four types of such vicinal surfaces can be distinguished. They are obtained by varying the angle $\alpha$ between the surface normal and the (001) direction. For increasing values of $\alpha$, one successively obtains:

- (001) vicinal surfaces;

- (111)-A vicinal surfaces, with (001) step microfacets;

- (11)-B vicinal surfaces, with (푼) step microfacets;

- (110) vicinal surfaces.

We note $\theta$ the miscut angle, i.e. the orientation between the surface plane and the nearest dense plane. All these surfaces can be indexed as ( $\mathrm{m} \mathrm{m} \mathrm{p).} \mathrm{Table} 1$ gives the geometrical details for all these surfaces. Atoms are labeled according to their distance to the step in the terrace plane. Thus atom $\mathrm{N}^{\circ} 1$ is the step edge atom, atom $\mathrm{N}^{\circ} 2$ is its nearest neighbor inside the terrace etc. For a vicinal with 10 atoms per terrace, atom $\mathrm{N}^{\circ} 10$ is the corner atom and 
atoms 11 to 20 are located just below the terrace plane. It is worth noting that this onedimensional procedure allows to reach every representative atomic position. An example of such procedure is given in figure 1 for a $\left(\begin{array}{lll}1 & 1 & 19\end{array}\right)$ surface.

\section{B. Calculations}

Atomic relaxations are computed at $T=0 \mathrm{~K}$, from the configuration of minimum energy. Atoms interact through a many-body empirical potential, derived from tight-binding considerations. This potential, hereafter referred to as the RGL potential ${ }^{13}$, is developed on the basis of a second-moment approximation (SMA) of the density of states for transition and noble metals.

The RGL potential energy is a sum over all atoms $i$ in the system:

$$
E_{p}=\sum_{i} E_{i}^{N}+E_{i}^{r e p}
$$

with

$$
E_{i}^{N}=-\sqrt{\sum_{j \neq i} \xi^{2} \exp \left(-2 q\left(r_{i j} / r_{0}-1\right)\right)}
$$

and

$$
E_{i}^{r e p}=\frac{1}{2} \sum_{j \neq i} A \exp \left(-p\left(r_{i j} / r_{0}-1\right)\right)
$$

where $r_{0}$ is the distance between nearest neighbors in the bulk at zero temperature and $r_{i j}$ the distance between atoms $i$ and $j$. For fcc metals, $r_{0}=a_{0} / \sqrt{2}$ where $a_{0}$ is the lattice constant. The band energy $E_{i}^{N}$ is a many-body attractive term while the repulsive energy $E_{i}^{\text {rep }}$ is written as a pairwise sum. The parameters $A, p, \xi$ and $q$ are determined by fitting the experimental values of the cohesive energy, the lattice parameter and the elastic constants. The parameters of the potential are taken from ref. ${ }^{14}$ for $\mathrm{Cu}$ and in ref. ${ }^{15}$ for the other metals and are given in Table 2. This potential is known to generally underestimate the surface 
energies and surface relaxations ${ }^{10,11}$. However, its simple form allows one to perform calculations over very large unit cells, which are up to now not possible by direct ab-initio methods. Moreover, our aim is not to give the exact description of vicinal surfaces of all fcc transition metals, but to determine how one can correlate the step relaxations and interactions with the main features of the interatomic potential. We will see that even the very simple form of the RGL potential gives rise to a variety of behavior for the different metals studied.

Calculations are done on slabs containing two free surfaces corresponding to $x y$ planes. Steps are oriented along $y$ and periodic boundary conditions are applied in the $x$ and $y$ directions. In the $z$ direction normal to the surface, the thickness of the slab is proportional to the interstep distance, in order to obtain negligible displacements at the center of the sample. In the $x$ direction, the sample contains one or two terraces. In the $z$ direction, taking for example (001) vicinals, the sample size goes from 10 (001) planes for (115) surfaces to 160 (001) planes for (1 1 79) surfaces.

The time step of the simulation is $10^{-14} \mathrm{~s}$ for $\mathrm{Pt}$ and $\mathrm{Au}$ and $5.10^{-15} \mathrm{~s}$ for $\mathrm{Ni}$ and $\mathrm{Cu}$. We have checked that these time steps give the required accuracy for the calculations. The number of time steps that are necessary for obtaining such good precision is proportional to the thickness of the sample. Thus, the duration of the simulations rapidly increases with the interstep distance.

\section{Atomic relaxations}

\section{A. Results}

The atomic relaxations are obtained by comparison with the crystallographic positions. In

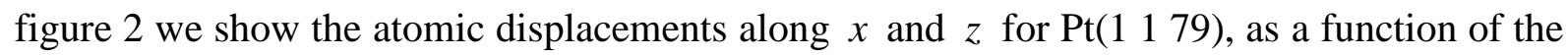
distance to the step. A mean relaxation of the terrace atoms, which corresponds to the relaxation of surface atoms for a nominal surface, is particularly visible in the $z$ direction. 
This mean relaxation $\vec{u}_{\text {Terrace }}$ can be also seen on the next (001) planes (below the terraces). The atomic relaxations on the nominal surface of a transition metal are due to the variation of the local density of states near the surface. A good order of magnitude for such modifications is given by the Fermi wavelength. For example, for copper, $\lambda_{F}(\mathrm{Cu})=0.46 \mathrm{~nm}$. Thus, these relaxations rapidly decay in the bulk.

On vicinal surfaces, in addition to this surface relaxation, there is a specific relaxation due to the steps, $\vec{u}_{\text {Step }}$. This contribution is particularly important at the vicinity of the steps, and especially for the step edge and corner atoms. On the (1 1179$)$ surface shown in figure 2, these atoms have respectively the labels 1 and 40 . Our calculations show that, except for $\mathrm{Ni}$, step edge atoms relax towards the inner terrace and towards the bulk, whereas corner atoms relax in the opposite direction. Such opposite relaxations can be seen in figure 2 for atoms near the step edge but deeper in the bulk. This gives rise to the periodic features appearing in the figure, with a period given by the number of atoms in the terrace. Whereas $\vec{u}_{\text {Terrace }}$ is rapidly negligible, $\vec{u}_{\text {Step }}$ has a much larger decay length.

For each crystal atom, $\vec{u}_{\text {Step }}$ can be obtained by subtracting to $\vec{u}$ the contribution $\vec{u}_{\text {Terrace }}$ corresponding to the atomic relaxations on a nominal surface. Thus, the specific contribution of the steps to the relaxations can be represented independently of the mean terrace relaxation. $\vec{u}_{\text {Step }}$ is presented in figure 3 for different vicinal surfaces with 10 atomic rows per terrace. As can be seen, the most relaxed atoms are located near the steps. For most cases, the step edge and corner atoms are the most relaxed atoms, with relaxations in the opposite direction. This can be seen for example on $\mathrm{Cu}\left(\begin{array}{lll}1 & 1 & 19\end{array}\right)$ or $\mathrm{Pt}(554)$ vicinals. However, for some cases, other atoms near the step display also a significant relaxation. This is especially the case for (111)B vicinals. For $\mathrm{Cu}(554)$, these two atoms relax in the same direction whereas atom $\mathrm{N}^{\circ} 9$ relaxes symmetrically. Nearly the same behavior can be observed for $\mathrm{Ni}(554)$. Thus, for these 
vicinals, regarding the atomic relaxations, the step is more likely a (110) microfacet for $\mathrm{Cu}$ and Ni but a (11' $)$ microfacet for Au and Pt.

For all cases, atomic relaxations propagate deeply into the bulk. A general feature that we always observe is the presence of vertices in the relaxation field, which are located below the middle of the terraces. They are particularly visible for Ni(554). As a consequence, deeply in the crystal, the relaxations along $x$ are inverted relatively to their direction near the surface.

In general, relaxations are smaller for Ni vicinals, except on (111)B vicinals, and higher for Pt and Au vicinals which display very similar relaxation fields.

\section{B. Dipolar model}

In the original description of Marchenko and Parshin (MP) ${ }^{8}$, the elastic displacements far from an isolated step on the surface of an isotropic medium are the same as those due to a line of elastic force dipoles on a flat surface. In the case of a $\mathrm{Cu}\left(\begin{array}{lll}1 & 1 & 19\end{array}\right)$ surface, the elastic displacements due to the steps have been shown to be the same as the elastic response of lines of dipoles on the surface ${ }^{14}$. There, the elastic response $\vec{u}_{\text {Dip }}(\vec{p})$ to a dipole $\vec{p}$ was calculated by QMD, with additional elastic forces on the atoms on which the dipole applies, a priori the step edge and corner atoms. In comparison with linear elasticity, atomistic calculations with our SMA potential have the advantage to take directly into account the local atomic structure, the real geometry of the surface and the variation of the elastic constants near the surface. On the other hand, the elastic forces need to apply on the atoms, thus the elastic dipole is necessarily an extended dipole, with a lever arm given by the positions of the step edge and step corner atoms. The elastic response $\vec{u}_{\text {Dip }}(\vec{p})$ is obtained by subtracting to the calculated displacements the initial value of the relaxations, $\vec{u}$. Note that, on contrary to MP model, the lever arm of the dipole is not in the surface plane, thus, the dipole is buried with one point of application of the force under the surface. 
For practically all cases, the atomic displacements $\vec{u}_{\text {Step }}$ are very well reproduced by the elastic response $\vec{u}_{\text {Dip }}(\vec{p})$ to a buried dipole located on the step edge and corner atoms. In our procedure, we obtain the value of $\vec{p}$ by a least-square fit procedure concerning all atoms except the atoms where the elastic forces are applied. The dipole can be split into two components: a "stretch" component which has no moment $p_{s}$ - we note it as positive when it corresponds to a contraction of the distance between step edge and corner atoms - and a torque component $p_{T}$, which has no dilatation - we note it as positive when it as the same orientation as $\vec{i} \wedge \vec{n}$ where $\vec{n}$ is the normal to the surface and $\vec{i}$ is a vector of the surface plane, perpendicular to the steps and oriented along the descending steps (see figure 4).

In figure 5, the comparison of the relaxation fields for various $\mathrm{Au}, \mathrm{Pt}, \mathrm{Ni}$ and $\mathrm{Cu}$ surfaces of different orientations is displayed. All surfaces have of the order of 20 atomic rows per terrace. The fits are for practically all the cases excellent, except for step edge and corner atoms on which the forces are directly applied. With only two adjustable parameters (value and orientation of the elastic force), the amplitude of the elastic displacement at the surface, their attenuation in the bulk and the general shape of the curves are well reproduced. Only the displacements computed on $\mathrm{Ni}\left(\begin{array}{lll}1 & 1 & 39\end{array}\right)$ and $\mathrm{Au}\left(\begin{array}{llll}4 & 41 & 1\end{array}\right)$ surfaces present a less good agreement. To appreciate the quality of the fits, it is worth considering the inset which concerns the relaxations for atoms far from the surface. The model reproduces not only the amplitude of the displacements, but also the detailed shape of the variations, i.e. the contribution of the different harmonics.

The values of the dipoles that allow such best fits are given in table 3. In the MP model ${ }^{8}$, the torque component of the dipole is given by the product of the step height by the surface stress of the flat surface $t h$. We have numerically computed this last value for all surfaces investigated. The comparison of $\tau$ with $p_{T}$ is given in table 3 . The comparison shows that in a great number of cases, the hypothesis of the MP model is true. However, for $\mathrm{Ni}(001)$, 
$\mathrm{Cu}(111) \mathrm{B}, \mathrm{Ni}(111) \mathrm{B}, \mathrm{Pt}(111) \mathrm{B}$ and $\mathrm{Au}(110)$, the agreement is poor. This cannot be attributed to a less good fit of the atomic relaxations by the elastic response to a dipole. For example, for $\mathrm{Ni}(1111$ 10), the fit is excellent for all atoms.

\section{Energies}

\section{A. Calculations}

The energies are obtained by taking the asymptotic limit of the system energy after relaxation. The surface energy $\gamma$ is obtained by comparison with a system without free surfaces. The step energy $\beta$ is derived from the surface energy through

$$
\gamma(\theta)=\gamma(0) \cos (\theta)+\frac{\beta}{h}|\sin (\theta)|
$$

where $h$ is the step height in the direction perpendicular to the nominal surface. As steps are not isolated, $\beta$ varies with the interstep distance. We note $\beta_{\text {int }}(\theta)$ the interaction energy between steps, defined by

$\beta(\theta)=\beta(0)+\beta_{\text {int }}(\theta)$

where $\beta(0)$ is the energy for creating an isolated step on the nominal surface. Of course, $\beta_{\text {int }}(\theta)$ is an effective interaction energy which takes into account both the variation of the crystallographic orientation of the surface when the miscut varies, that can modify the selfenergy of the step, and the variation of the interstep distances that changes the step interactions.

It has been shown that in the case of elastic interactions between steps, $\beta_{\text {int }}(\theta)$ is inversely proportional to $d^{2} \cdot{ }^{8,12}$ At short distances, corrections may be necessary. In all cases, we have fitted $\beta_{\text {int }}(\theta)$ with a two-parameter equation:

$$
\beta_{\text {int }}(\theta)=\frac{A}{d^{2}}+\frac{B}{d^{3}}
$$


$A$ is often expressed in eV.A. For the comparison of vicinals with different types of terraces, we find more convenient to express $A$ in meV/at. In that case, $d$ is the number of $[1 \overline{1} 0]$ rows between the steps and the energy is given per atom along the step edge.

Using a semi-empirical potential with a cut-off length in direct space for the energy calculations allows to study only the elastic part of the interactions. In our model, the interactions are limited to the fifth neighbours. Beyond this cut-off, interactions are purely elastic. On the contrary, ab-initio electronic calculations in phase space take into account all contributions to the energy and the elastic contribution cannot be easily extracted.

In order to obtain a value of $A$ with a correct precision, we have calculated the step energies up to interstep distances of $40[1 \overline{1} 0]$ rows. At such distances $\beta_{\text {int }}(\theta)$ can be as small as few $\mu \mathrm{eV}$. It may seem paradoxical to calculate this energy with such a precision, since the value of the surface energies are known to be strongly underestimate. However, we are only interested in the accurate description of the elastic interactions and their relation to the features of the steps. We believe, the elastic interactions are described well by the chosen interatomic potentials, which were fitted to the elastic constants of the material. Figure 6 displays the variation of $\beta_{\text {int }}$ with interstep distance for all surfaces studied. It is always possible to adjust the variation of $\beta_{\text {int }}$ with Eq. (6). The fits are very good and show that the step interactions are really proportional to $1 / d^{2}$. The small deviations from the fit that can be observed at large interstep distances are due to numerical errors and to the uncertainties related to the subtraction of $\beta(0)$. First order component, i.e. inversely proportional to the interstep distance where always found negligible, on contrary to some assertions found in the literature. $^{16}$

\section{B. Results}


The values obtained for the surface and step energies and for the step interactions are given in table 4 . The calculations show that the step energy varies considerably from one metal to another and from one orientation to another. The highest differences between the metals are observed on (111) vicinal surfaces, whereas the lowest differences are seen on (110) vicinal surfaces. Small values of the step energy are obtained when the surface energy of the step microfacet is significantly smaller than the surface energy of the nominal surface corresponding to the terrace orientation. This is the case for $\mathrm{Au}$ and $\operatorname{Pt}(110)$ vicinals for which the step energy is negative, the surfaces are thus unstable. It is worth noting that during the simulation, the surface does not reconstruct since the reconstruction process is thermally activated. Experimentally, one observes a $(2 x 1)$ surface reconstruction of missing row nature for these surfaces. This reconstruction has also been shown to be energetically favored when calculating the system energy with a SMA potential ${ }^{17}$.

A good estimate of the step energies can be simply derived by considering that the step is a microfacet whose energy is given by the surface energy of the corresponding nominal surface. This is equivalent to simple models of the step energy based on the coordination of the atoms near the step. ${ }^{18}$

One thus obtains, after subtracting the adequate contribution of the terraces:

- for $(001)$ vicinals : $\beta=\frac{a_{0}^{2}}{4}\left(\gamma_{(111)} \sqrt{3}-\gamma_{(001)}\right)$

- for (111)A vicinals : $\beta=\frac{a_{0}^{2}}{2}\left(\gamma_{(001)}-\frac{\gamma_{(111)}}{\sqrt{3}}\right)$

- for (111)B vicinals : $\beta=\frac{a_{0}^{2}}{2 \sqrt{3}}\left(\gamma_{(111)}\right)$

- for (110) vicinals : $\beta=\frac{a_{0}^{2}}{\sqrt{8}}\left(\frac{\sqrt{3}}{2} \gamma_{(111)}-\gamma_{(110)}\right)$ 
Table 4 shows that this approximation is very good, except for $\mathrm{Ni}$, for which the step energies are overestimated. Note that it still gives negative step energies for $\mathrm{Au}$ and $\operatorname{Pt}(110)$ vicinals. Only few measurements of the step energy have been done on $\mathrm{Cu}$ vicinal surfaces. Our values are in relatively good agreement with the experimental values, indicating $\beta(0)=220 \mathrm{meV} /$ at for $\mathrm{Cu}(001)$ steps and $\beta(0)$ ranging from 220 to $450 \mathrm{meV} /$ at for $\mathrm{Cu}(111)$ steps ${ }^{19}$.

The step interaction energies also depend on the geometry of the surface and of the metal studied. They are always repulsive $(A>0)$. The comparison with the elastic displacements shows that high step interaction energies correspond to high elastic displacements. Concerning the variations with the metal studied, the higher $A$ coefficients are obtained for $\mathrm{Au}$ and $\mathrm{Pt}$, whereas $\mathrm{Ni}$ surfaces give rise to small interactions between steps, except for $\mathrm{Ni}(111) \mathrm{B}$ vicinals. Concerning the step geometry, low interaction energies are always found for (110) vicinals whereas high values are obtained on (111) vicinals, except for $\mathrm{Cu}$ where $A$ is higher on $\mathrm{Cu}(001)$ vicinals. It is very surprising to obtain very different interactions for Ni(111)A or B vicinals. $A_{(11) \mathrm{B}}$ is more than one order of magnitude higher than $A_{(111) \mathrm{A}}$. This is not the case for the other metals for which $A_{(111) \mathrm{A}}$ and $A_{(111) \mathrm{B}}$ are similar.

\section{Comparison with linear elasticity}

In section 3, we have compared the atomic relaxations due to steps on a vicinal surface to the elastic displacements due to lines of elastic dipoles, both computed by atomistic calculations. This approach was fruitfull in most cases, but is limited for two main reasons:

- first, it does not allow to test various force distributions since, in the atomistic calculations, forces need to apply on atoms;

- second, it does not allow to easily derive the step interaction energy. 
On the contrary, linear elasticity theory allows to calculate the displacement due to complex force distributions and to extract interaction energies. It has been shown that the elastic displacements created by topological defects could always be described, in the frame of linear elasticity, by an adequate distribution of forces near the defect, provided that the atoms for which the displacement is studied are far enough from the defect ${ }^{20}$.

Landau and Lifshitz have calculated the elastic response to a point force at the surface of a semi-infinite medium $^{21}$. In the case of a periodic distribution of forces, analytical calculations taking into account the crystalline anisotropy can be performed. For particular distributions, the harmonic resummation can even be achieved. It is for example the case of lines of forces with a lorentzian broadening.

\section{A. Displacements}

For all surfaces studied by atomistic calculations, we have compared the atomic displacements calculated with the analytical results of the linear elasticity theory. For this purpose, we have tried to optimize the force distribution near the surface in order to reproduce as correctly as possible the atomic displacements. We have tested two different distributions. Note that for mechanical equilibrium, they all need to be dipolar. For practical reasons of resummation, they are all periodic distributions of lines of point forces, with a lorentzian broadening along the $y$ direction, of width $a_{c}$. The different distributions tested are:

- a distribution of "geometric" buried dipoles. Each step is modeled by an extended dipole consisting of two lines of opposite forces. The first line is at the step edge, and the second line at the step corner.

- a distribution of point dipoles buried below the surface. The orientation of the lever arm of the dipoles, their depth and their position along $y$ with respect to the steps are free parameters. 
The details of the anisotropic linear elasticity (ALE) calculations are given in ref. ${ }^{22}$

\section{1. "geometric buried dipole" distribution}

In that case, there are three free parameters: the value of the force applied, its orientation and the cut-off length of the broadening $a_{c}$. The fitting procedure is a least-square fit taking into account all atoms except step edge and step corner atoms. We have observed that the elastic response calculated in that way could be quite different from the elastic response given by atomistic calculations. Generally, the agreement is worse. For some cases, i.e. Ni(111) and $\mathrm{Cu}(111) \mathrm{B}$ vicinals, a correct agreement is not possible. The values of the dipoles that allow the best fits are given in table 5 .

In all cases, the value of the torque component of the elastic dipole found by ALE is higher than the value found by atomistic calculations. The differences observed are probably due to the modification of the elastic constants near the surface and near the step. The low coordination of step edge atoms gives rise to a lowering of the Young modulus in comparison with bulk atoms. The value of the forces that have to be applied on these atoms in order to obtain the wanted relaxations are thus lower if this modification of the elastic constant is taken into account (i.e. in the atomistic calculations) than if it is not the case (i.e. for ALE calculations). The differences could also be due to second order corrections to the linear elasticity theory.

When the terrace length varies, the value of the elastic dipoles giving the best fit also varies. This variation for $\mathrm{Cu}$ and $\mathrm{Pt}(111) \mathrm{A}$ vicinals in given in figure 7. In most of the cases, the stretch component varies whereas the torque component remains constant. This is due to the fact that the elastic displacements due to stretch dipoles are lower than those due to torque 
dipoles of same value. The observed variation is thus mainly to be related to the uncertainty on the value of $p_{\mathrm{S}}$.

One of the assets of ALE calculations is to give directly the values of the step interactions. The basis of the energy calculation is that we first determine a force distribution that allows to correctly describe the displacement field. Thus, it is fundamental to well reproduce the atomic relaxations. In order to go further, we have thus tested another force distribution, presenting more adjustable parameters.

\section{2. "Point dipole" distribution buried under the surface}

This force distribution derives from the MP Model, but with four differences: the lorentzian broadening already discussed, the orientation of the lever arm, not necessarily parallel to the surface, the fact that the dipoles are buried under the surface and the crystalline anisotropy of the material. The point dipole is the limit of a buried dipole with a vanishing distance between the points of application of the forces. But, on contrary to the previous paragraph, the lever arm orientation and the position of the lines of dipoles are free parameters.

When trying to adjust the SMA relaxations with ALE calculations using dipolar distributions, one observes that far from the defect, the elastic displacements can be adjusted by several combinations of parameters (position, lever arm orientation, cut-off length and force value). In figure 8 is displayed the variation of the $\chi^{2}$ for the fit of the atomic relaxations on $\mathrm{Cu}, \mathrm{Ni}, \mathrm{Pt}$ and $\mathrm{Au}(15,15,16)$ surfaces as function of $\Omega$, the lever arm orientation of the elastic dipoles. For each $\Omega$ value, the free parameters are the force value and orientation, the position of the dipole lines and the cut-of length. As can be seen, a good fit is possible for different $\Omega$ values. In figure 8 , one also observes that there exist some directions for which it is impossible to obtain a correct adjustment. For Ni(15 15 16), the 
forbidden direction is close to the step microfacet direction. This explains why it was not possible to obtain a good fit using the "geometric" buried dipole model. An example of fit by the two models is given in figure 9 for a $\mathrm{Cu}\left(\begin{array}{lll}1 & 1 & 79\end{array}\right)$ surface. Whereas the fit is not perfect with the "geometrical buried dipole" model, the fit is excellent with the point dipole model. It is not surprising since the number of free parameters is higher (6 instead of 3 ).

The values of the dipoles that allow the best fit with the atomic relaxations are given in table 6. A comparison with the results obtained with the "geometrical buried dipole" model can be made. The value of the torque component $p_{T}$ is roughly the same, whereas the value of the stretch component $p_{S}$ is considerably modified. This is related to the fact that stretch point dipoles give rise to much lower displacements than torque point dipoles of the same intensity. In the fitting procedure, the uncertainty on the value of $p_{S}$ is thus much higher than the uncertainty on the value of $p_{T}$.

\section{B. Interaction energy}

In the case of a dipolar force distribution, it is possible to calculate analytically the elastic interaction energy from the dipole value $\mathrm{e}^{22}$. The elastic energy per dipole line takes the form:

$$
\beta(d)=\beta_{0}+\frac{A}{d^{2}}+O\left(\frac{1}{d^{4}}\right)
$$

where the first term is the elastic energy of an isolated line and the second term, the interaction energy between lines. Contrary to SMA calculations, it is not possible to discuss the first term which is associated with the energy of an isolated step. It has no physical meaning. First, it cannot be equal to the step energy since it does not account for the local cost of the coordination reduction of atoms near the steps. Second, it corresponds to the result of the calculation of the elastic energy in a domain where linear elasticity does not apply. For 
example, when the distance between the points of application of the forces get close to zero, $\beta_{0}$ becomes infinite; the crystal present a singularity at the dipole position.

The coefficients $A$ are given in table 7 for the "geometric buried dipole" and "buried point dipole" distributions that allow the best fits with SMA calculations. Since elastic energy directly derives from the atomic deformations, two force distributions that give the same deformations far from their application points also give similar values for the elastic interaction energy. In the case where the fit is not correct for the atomic relaxations, for example for $\mathrm{Ni}(1616$ 15) when using the "geometrical buried dipole" model, the values found for the two distributions are very different.

The comparison between the coefficients found by ALE for large interstep distances and by SMA from Eq. (6) is given in figure 10. We have not given the values obtained by ALE in the case of the buried dipole model when the fit was poor (for three cases). A good agreement is found on the whole range of values. The standard deviation between the values found by ALE and SMA is of the order of $28 \%$ when considering "geometrical buried dipoles" in the elasticity calculations, and $18 \%$ when considering "buried point dipoles". This is not surprising since the fits of the relaxations are better when using buried point dipoles. In that case, depending on the surface studied, the values obtained by ALE are either higher or lower than the values obtained by SMA. The most important differences are observed for $\mathrm{Ni}(6161$ 1) for which ALE calculations with buried point dipoles indicate a value $40 \%$ higher than QMD calculations, and for $\mathrm{Cu}(1515$ 16) for which the same ALE calculations indicate a value $70 \%$ lower than SMA calculations.

\section{Discussion}


Our comparisons show that the calculation of the displacements due to a dipolar force distribution in the framework of the linear elasticity allows to reproduce correctly the relaxations found by SMA. The force distribution that allows a good fit far from the step is not unique. Good fits are obtained for different dipole orientations, but two distributions that give a good agreement for the relaxations also give similar values for the step interaction energy.

Small differences are observed between the values of the step interaction energy found by ALE and by SMA. It is difficult to explain their origin. A first explanation could be due to the variation of the elastic self-energy of the step which is function of the step orientation: when the surface orientation is rotated, the directions of the lever arm of the elastic dipole and the direction of the elastic forces also rotate. Such effect would lead to a first order correction to the step elastic energy $\beta_{0}$ with the miscut, and thus to an additional $1 / d$ coefficient in Eqs. 6 and 7. We have already mentioned that it is not possible to obtain this coefficient by ALE since we cannot calculate a real value of the elastic self-energy of a step. Moreover, our SMA results show that such a coefficient is always negligible and cannot explain the differences observed. These differences could be due to the variation of elastic constants near the surface and also to the non-linearity of the elastic deformations close to the step. This would affect the step elastic interactions. However, there is no obvious relation between the differences observed and these parameters. Thus, the differences observed are probably due to the uncertainties on the values of $A$ determined by SMA using Eq. (6) and by ALE.

The comparison between the different surfaces of the different transition metals studied shows that the step interactions are related to the surface stress. The step interaction energy is mainly given by the value of the torque component of the dipoles, $p_{T}$, which is roughly given by the product of the surface stress by the step height. As shown by Olivier et al. ${ }^{17}$, the variations of surface stress, from one surface to another, are highly related to the attractive 
interaction between atoms through the d-band electrons. Due to the lower coordination at the surface, the number of electrons in the d-band is lowered, the electronic repulsion is lowered and the binding is stronger. In the RGL model, the range of the attractive interactions is given by the $q$ parameter. With the chosen parameterization for the potential, $q$ is higher for $\mathrm{Au}$ and $\mathrm{Pt}$ than for $\mathrm{Cu}$ and $\mathrm{Ni}$ for which $q$ is very small. The first neighbors have thus a higher contribution in the case of $\mathrm{Au}$ and $\mathrm{Pt}$, than in the case $\mathrm{Cu}$ and $\mathrm{Ni}$.

The influence of the geometry of the surface on the interactions can also be discussed. The differences between the metals are higher on (111) surfaces than on (110) surfaces. On (111) surfaces, which are the most compact surfaces for FCC crystals, and for which the interrow distance is the smallest, there are 6 first neighbors in the surface plane, and 3 first neighbors in the other planes: for $\mathrm{Pt}(111)$ and $\mathrm{Au}(111)$, the in-plane bindings are strengthened, leading to high surface stress. On (110) surfaces, which are the less compact dense surfaces, there are only 2 first neighbors in the surface plane and 5 first neighbors in the other planes. For $\operatorname{Pt}(110)$ and $\mathrm{Au}(110)$, the surface stress is small, but the interplanar relaxations are high. For $\mathrm{Cu}$ and especially $\mathrm{Ni}$, the next neighbors have a greater importance and the effects previously mentioned are lowered.

These simple considerations allow to understand all the behaviors observed, except the case of $\mathrm{Ni}(111)$ vicinals. The reason why (111)B vicinals display important elastic displacements in opposite to (111)A vicinals remains unexplained.

\section{Conclusion}

The comparison between SMA and ALE shows that, in a first order approximation, a step on a vicinal surface is equivalent to a line of force dipoles on a flat surface. The force distribution that allows to reproduce the atomic relaxations far from the step is not unique. 
Buried point dipoles with lever arm oblique to the surface plane give a very good agreement. Using this simple force distribution, it is possible to fit the atomic relaxations and to obtain, with a good precision, the elastic step interaction energy. The knowledge of the atomic relaxations is thus sufficient for determining precisely the elastic interaction energy. This is not trivial since the interaction energy is only a second order correction to the total elastic energy, whose main contribution is the step self-energy. This demonstrates that it is possible to extract correct values for the step interactions from the measurements by grazing incidence $\mathrm{X}$-ray diffraction of the atomic relaxations on vicinal surfaces ${ }^{10,11}$.

Tables

\begin{tabular}{|l|c|c|c|c|}
\hline terrace orientation & $(001)$ & $(111) \mathrm{A}$ & $(111) \mathrm{B}$ & $(110)$ \\
\hline Step microfacet & $(111)$ & $(001)$ & $(\overline{1} \overline{1} 1)$ & $(111)$ \\
\hline Surface orientation & $(1,1,2 n+1)$ & $(n-1, n-1, n+1)$ & $(n+1, n+1, n-1)$ & $(2 n+1,2 n+1,1)$ \\
\hline Number of atoms & $n+1$ & $n$ & $n+1$ & $n+1$ \\
per terrace & $\frac{1}{2 \sin (\alpha)}$ & $\frac{1}{\sin (\alpha)-\cos (\alpha) \sqrt{2}}$ & $\frac{1}{\sin (\alpha)-\cos (\alpha) \sqrt{2}}$ & $\frac{1}{2 \sqrt{2} \cos (\alpha)}$ \\
\hline Interstep distance $d$ & $\frac{1}{2}$ & $\frac{1}{\sqrt{3}}$ & $\frac{1}{\sqrt{3}}$ & $\frac{1}{2 \sqrt{2}}$ \\
\hline Step height & $\frac{1}{2}$ & & & \\
\hline
\end{tabular}

Table 1. Geometrical parameters for the vicinal surfaces of fcc crystals with dense steps. Values are given in unit of the lattice constant. $\alpha$ is the angle between the surface orientation and (001). 


\begin{tabular}{|l|l|l|l|l|}
\hline & $\mathrm{Ni}$ & $\mathrm{Cu}$ & $\mathrm{Pt}$ & $\mathrm{Au}$ \\
\hline$A(\mathrm{eV})$ & 0.0752 & 0.18975 & 0.595 & 0.4122 \\
\hline$\xi(\mathrm{eV})$ & 1.070 & 1.2603 & 2.695 & 1.790 \\
\hline$p$ & 16.999 & 10.550 & 10.612 & 10.229 \\
\hline$q$ & 1.189 & 2.43 & 4.004 & 4.036 \\
\hline$a_{0}(\AA)$ & 3.523 & 3.61 & 3.924 & 4.079 \\
\hline
\end{tabular}

Table 2. Parameters of the RGL potential described in Eqs. 1-3 for the different metals studied. 


\begin{tabular}{|l|l|l|l|l|}
\hline & $\mathrm{Ni}$ & $\mathrm{Cu}$ & $\mathrm{Pt}$ & $\mathrm{Au}$ \\
\hline$p_{S(1139)}$ & 3.53 & 1.61 & 3.41 & 2.39 \\
\hline$p_{T(1139)}$ & $1.35(2.30)$ & $2.10(2.37)$ & $3.97(4.06)$ & $2.35(2.42)$ \\
\hline$p_{S(101011)}$ & 9.02 & 3.57 & 1.38 & 0.50 \\
\hline$p_{T(101011)}$ & $0.37(0.45)$ & $1.65(1.85)$ & $5.44(5.34)$ & $3.36(3.27)$ \\
\hline$p_{S(111110)}$ & 2.62 & 1.23 & -0.04 & 1.88 \\
\hline$p_{T(111110)}$ & $-1.62(0.45)$ & $0.40(1.85)$ & $3.80(5.34)$ & $3.27(3.27)$ \\
\hline$p_{S(41411)}$ & -1.17 & 0.24 & 7.17 & 4.79 \\
\hline$p_{T(41411)}$ & $1.91(2.02)$ & $1.47(1.40)$ & $1.35(1.46)$ & $0.76(0.84)$ \\
\hline
\end{tabular}

Table 3. Values of the elastic dipoles $\left(10^{-10} \mathrm{~N}\right)$ that allow the best fits with the atomic displacements on various vicinal surfaces. The light gray cells indicate that the agreement is not perfect. The values in brackets are the product of the step height by the surface stress (same units). 


\begin{tabular}{|l|l|l|l|l|}
\hline & $\mathrm{Ni}$ & $\mathrm{Cu}$ & $\mathrm{Pt}$ & $\mathrm{uu}$ \\
\hline$\gamma_{(001)}$ & 2613.72 & 1272.94 & 1064.01 & 577.89 \\
\hline$\beta_{(001)}$ & 273.4 & 149.3 & 114.8 & 62.8 \\
\hline$\beta_{(001) \text { Microfact }}$ & 363 & 156 & 122 & 71 \\
\hline$A_{(001)}$ & 7.4 & 29.3 & 59.5 & 38.9 \\
\hline$\gamma_{(111)}$ & 2588.61 & 1176.88 & 907.79 & 491.30 \\
\hline$\beta_{(111) \mathrm{A}}$ & 342.5 & 237.8 & 245.6 & 139.3 \\
\hline$\beta_{(111) \mathrm{A} \text { Microfacet }}$ & 434 & 242 & 260 & 153 \\
\hline$A_{(111) \mathrm{A}}$ & 12.8 & 14.6 & 129.3 & 90.6 \\
\hline$\beta_{(111) \mathrm{B}}$ & 326.6 & 239.3 & 237.9 & 132.5 \\
\hline$\beta_{(111) \mathrm{B} \text { Microfact }}$ & 580 & 277 & 252 & 148 \\
\hline$A_{(111) \mathrm{B}}$ & 212.5 & 8.9 & 229.8 & 160.9 \\
\hline$\gamma_{(110)}$ & 2777.05 & 1379.95 & 1138.01 & 614.14 \\
\hline$\beta_{(110)}$ & 84.1 & 16.8 & -24.6 & -16.5 \\
\hline$\beta_{(110) \text { Microfacet }}$ & 108 & 18 & -9 & -5 \\
\hline$A_{(110)}$ & 2.2 & 2.0 & 1.2 & 0.9 \\
\hline \hline
\end{tabular}

Table 4. Surface energies $\gamma\left(\mathrm{mJm}^{-2}\right)$ of the nominal surfaces, step energies $\beta$ (meV/at), step energies obtained from the microfacet decomposition and step interaction coefficient $A$ (meV/at) for the different surfaces studied. 


\begin{tabular}{|l|l|l|l|l|}
\hline & $\mathrm{Ni}$ & $\mathrm{Cu}$ & $\mathrm{Pt}$ & $\mathrm{Au}$ \\
\hline \hline$p_{S(1179)}$ & 0.78 & 4.01 & 12.76 & 7.79 \\
\hline$p_{T(1179)}$ & 2.42 & 2.65 & 4.19 & 2.45 \\
\hline$p_{S(151516)}$ & 11.50 & 2.21 & 0.03 & 0.15 \\
\hline$p_{T(151516)}$ & -0.18 & 1.95 & 6.12 & 3.69 \\
\hline$p_{S(161615)}$ & 15.03 & 4.12 & 9.29 & 6.71 \\
\hline$p_{T(161615)}$ & -2.21 & 1.30 & 6.21 & 3.71 \\
\hline \hline$p_{S(61611)}$ & -0.43 & 0.43 & 4.21 & 2.82 \\
\hline$p_{T(61611)}$ & 1.96 & 1.52 & 1.52 & 0.87 \\
\hline \hline
\end{tabular}

Table 5. Values of the "geometric buried dipoles" $\left(10^{-10} \mathrm{~N}\right)$ that allow the best fits of the SMA atomic relaxations on various vicinal surfaces. The light gray cells indicate that the fit is not excellent, whereas the dark gray cells indicate that a correct fit is not possible. 


\begin{tabular}{|l|l|l|l|l|}
\hline & $\mathrm{Ni}$ & $\mathrm{Cu}$ & $\mathrm{Pt}$ & $\mathrm{Au}$ \\
\hline \hline$p_{S(1179)}$ & 0.91 & 5.54 & -9.87 & -5.92 \\
\hline$p_{T(1179)}$ & 2.22 & 2.18 & 3.43 & 2.07 \\
\hline \hline$p_{S(151516)}$ & 10.05 & 3.72 & -7.54 & -4.59 \\
\hline$p_{T(151516)}$ & 0.83 & 1.69 & 4.31 & 2.64 \\
\hline \hline$p_{S(161615)}$ & 19.49 & -2.22 & 17.30 & 10.13 \\
\hline$p_{T(161615)}$ & 1.39 & 2.09 & 4.81 & 2.99 \\
\hline \hline$p_{S(61611)}$ & 5.37 & -4.00 & 2.59 & 1.67 \\
\hline$p_{T(61611)}$ & 2.09 & 1.36 & 1.38 & 0.79 \\
\hline \hline
\end{tabular}

Table 6. Values of the buried point dipoles $\left(10^{-10} \mathrm{~N}\right)$ that allow the best fits of the SMA atomic relaxations on various vicinal surfaces. The agreement is always excellent. 


\begin{tabular}{||l||l|l||l|c||c|c||c|c||}
\hline \multirow{2}{*}{} & \multicolumn{3}{|c||}{ Ni } & \multicolumn{3}{c|}{ Cu } & \multicolumn{2}{c||}{ Pt } \\
\cline { 2 - 9 } & GBD & \multicolumn{1}{|c|}{ PD } & GBD & PD & GBD & PD & GBD & PD \\
\hline \hline$A_{(1179)}$ & 9.2 & 5.6 & 22.4 & 26.4 & 56.1 & 57.0 & 39.6 & 38.7 \\
\hline$A_{(151516)}$ & 0.12 & 24.8 & 10.1 & 10.1 & 67.4 & 124.4 & 47.7 & 88.7 \\
\hline$A_{(161615)}$ & 16.8 & 157.2 & 6.5 & 9.9 & 143.3 & 201.7 & 111.3 & 144.6 \\
\hline$A_{(61611)}$ & 3.0 & 2.4 & 2.4 & 2.1 & 1.5 & 1.5 & 1.1 & 0.6 \\
\hline
\end{tabular}

Table 7. Value of the interaction energy coefficient $A$ (meV/at) for "geometrical buried dipoles" (GBD) and for point dipoles (PD). Dark gray cells indicate that a correct fit of the relaxations was not possible with the chosen force distribution. 


\section{Figures}
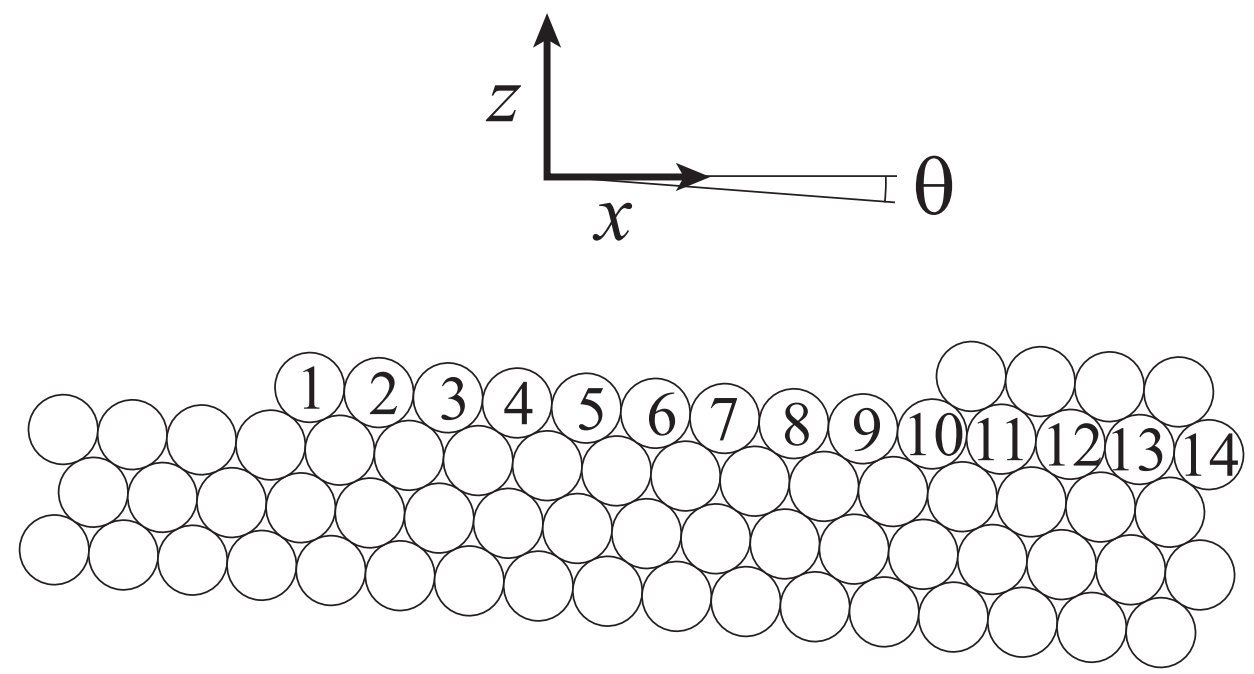

Figure 1. Schematic of a vicinal surface (in this example, the (lllll 119 ) surface), with indication of the way how the atoms are labeled. $\theta$ is the miscut angle. 


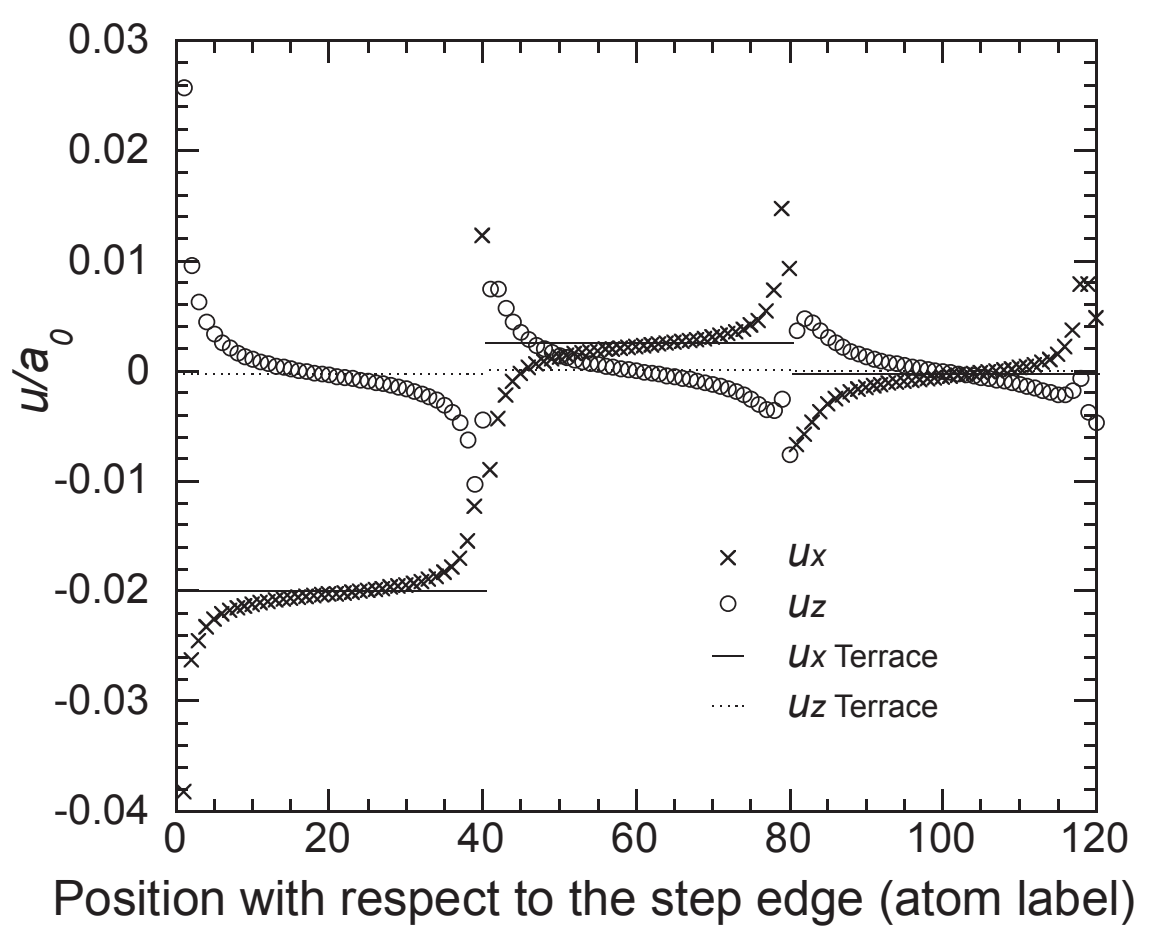

Figure 2. Atomic displacements $\vec{u}$ along $x$ and $z$ for $\operatorname{Pt}\left(\begin{array}{ll}1 & 179\end{array}\right)$, as a function of the distance to the step along the $\left[\begin{array}{ll}1 & 1\end{array}\right]$ axis, and specific contribution of the terrace relaxation $\vec{u}_{\text {Terrace }}$. The $x$ axis gives the label of the atom. $a_{0}$ is the lattice constant. 
$\mathrm{Ni}(554)$
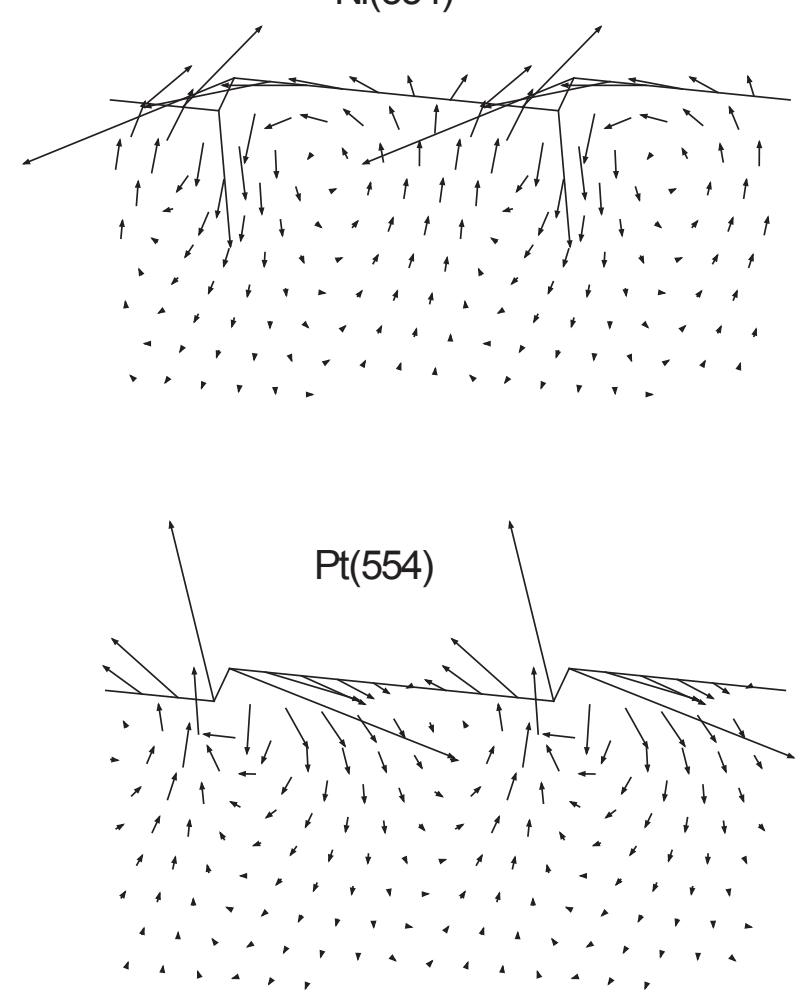

$\mathrm{Cu}(554)$
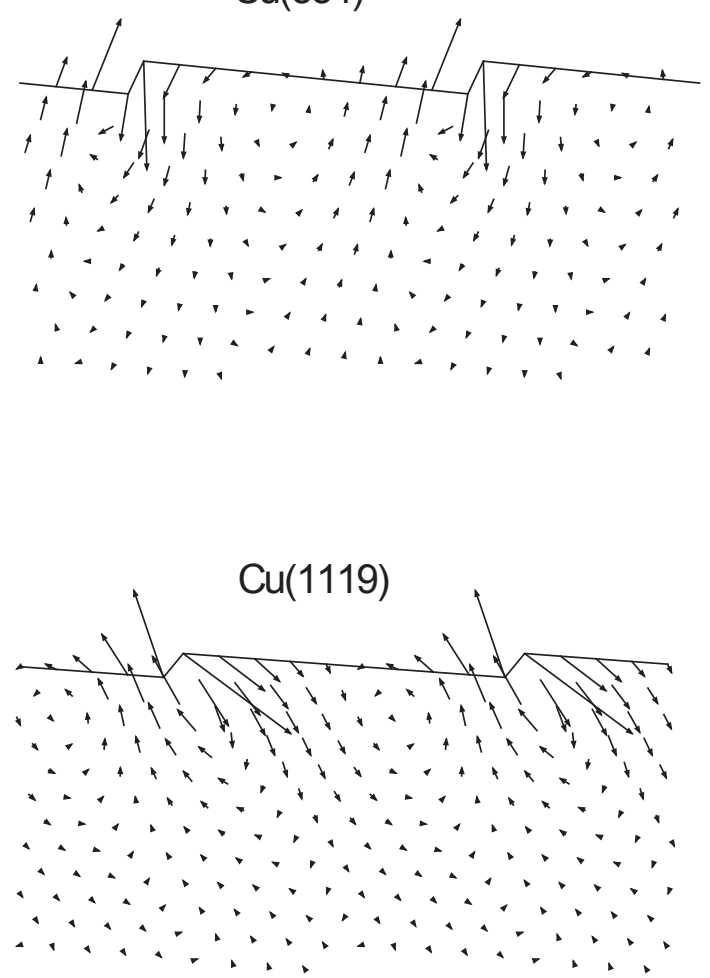

Figure 3. Contribution $\vec{u}_{\text {Step }}$ to the atomic relaxations for different vicinal surfaces. The amplitude of the relaxations is amplified by a factor of 100 . 


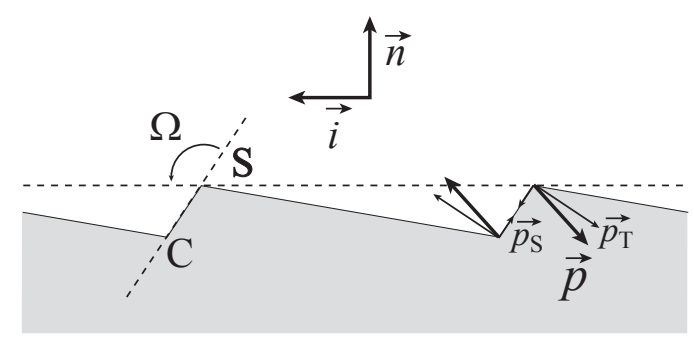

Figure 4. Schematic of a vicinal surface showing the force distribution corresponding to the dipole components $p_{\mathrm{S}}$ and $p_{\mathrm{T}}$ in the case of a "geometrical buried" dipole. $\Omega$ is the level arm orientation; $\mathrm{S}$ and $\mathrm{C}$ are the step edge and corner atom positions. In the example chosen, $p_{\mathrm{S}}$ and $p_{\mathrm{T}}$ are positive. 

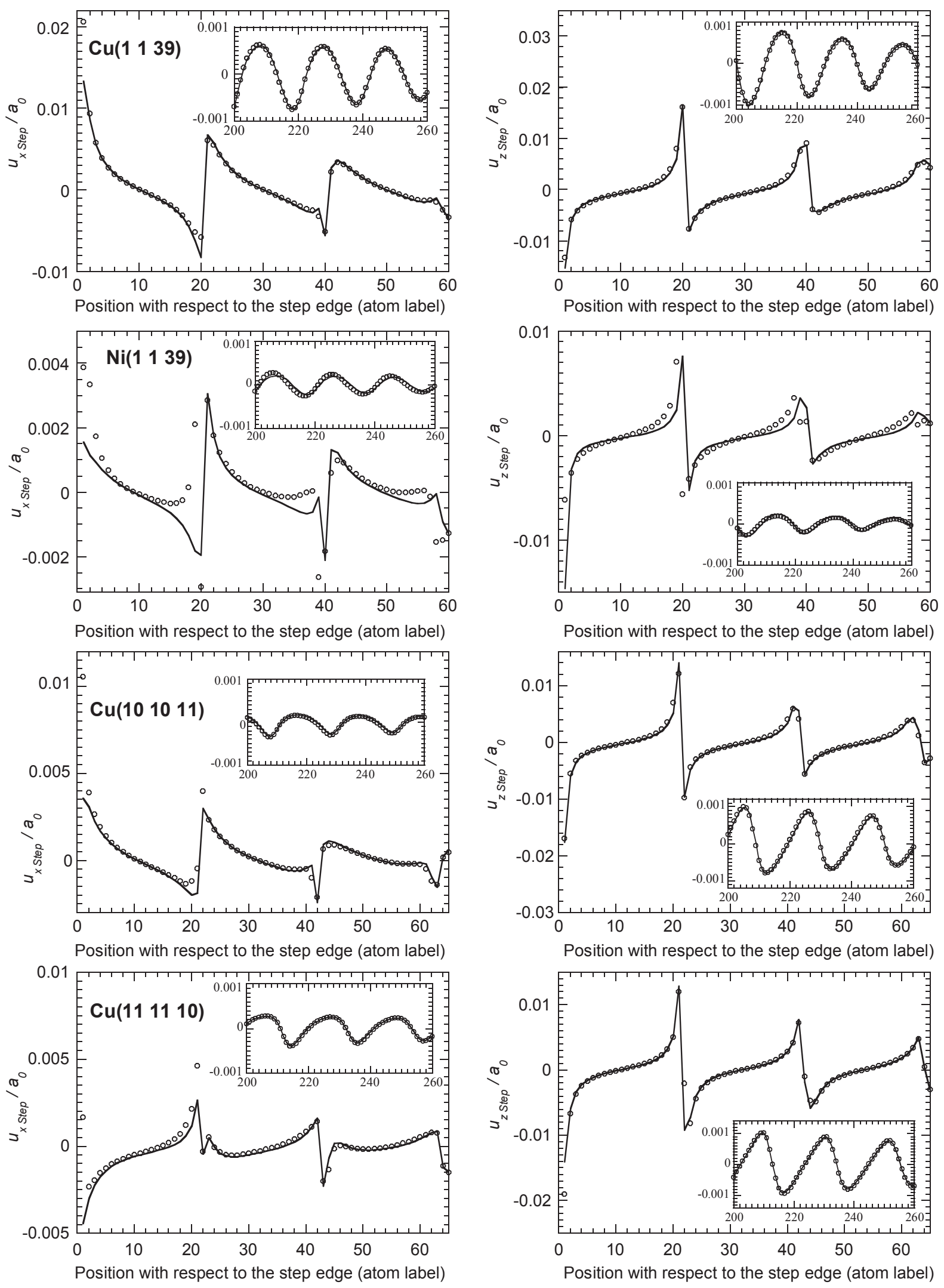

Figure 5. Comparison of the relaxation fields $\vec{u}_{\text {Step }}$ and $\vec{u}_{\text {Dip }}(\vec{p})$ calculated by SMA for various surfaces of $\mathrm{Au}, \mathrm{Pt}, \mathrm{Cu}, \mathrm{Ni}$. Dots: $\vec{u}_{\text {Step }}$. Lines: $\vec{u}_{\text {Dip }}(\vec{p})$. 

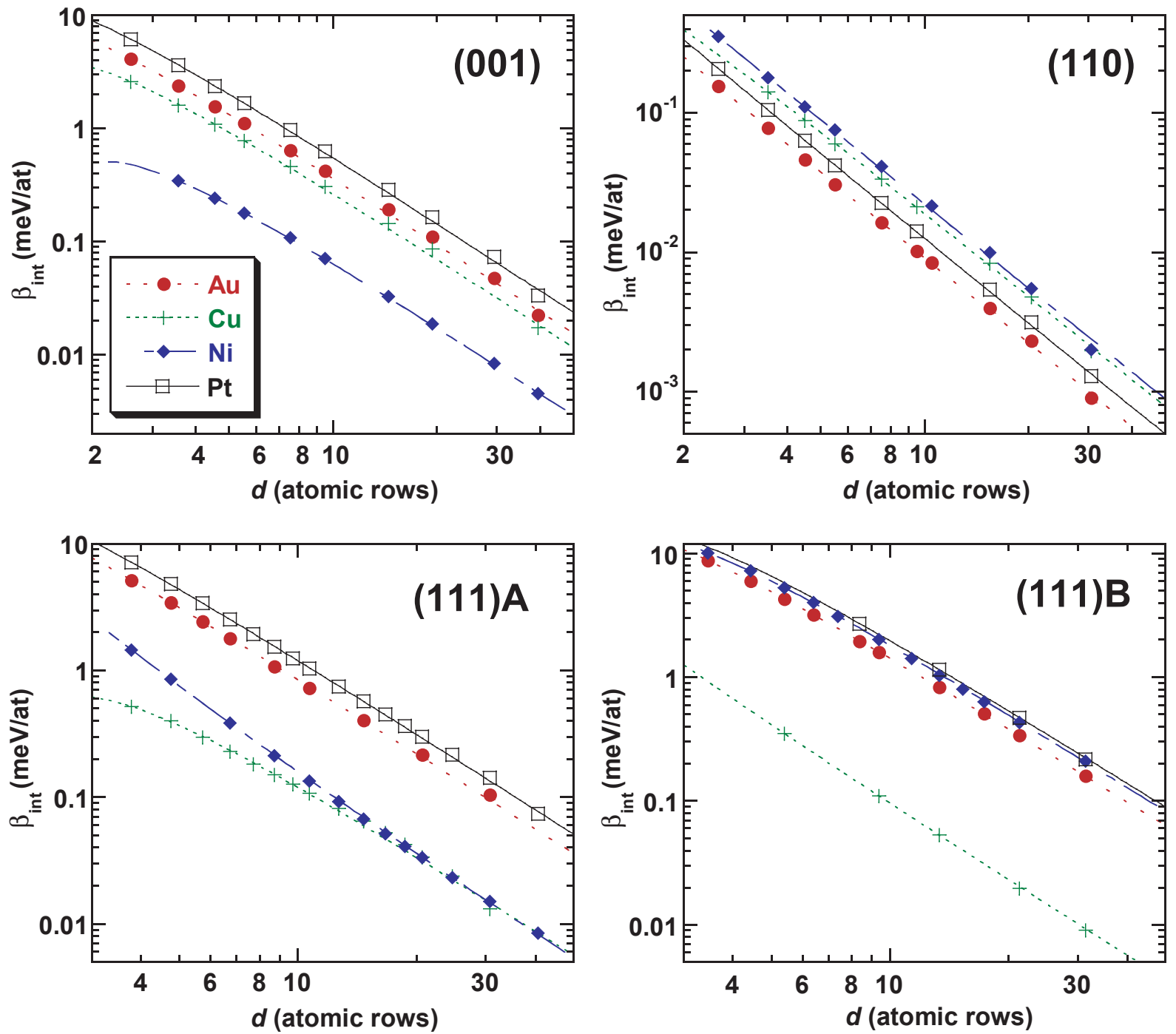

Figure 6 (color online). Variation of the step interaction energy $\beta_{\text {int }}(\theta)$ with interstep distance (symbols) together with fits with Eq. 6 (lines). Red dots: $\mathrm{Au}$; green crosses: $\mathrm{Cu}$; blue diamonds: Ni; black squares: Pt. 

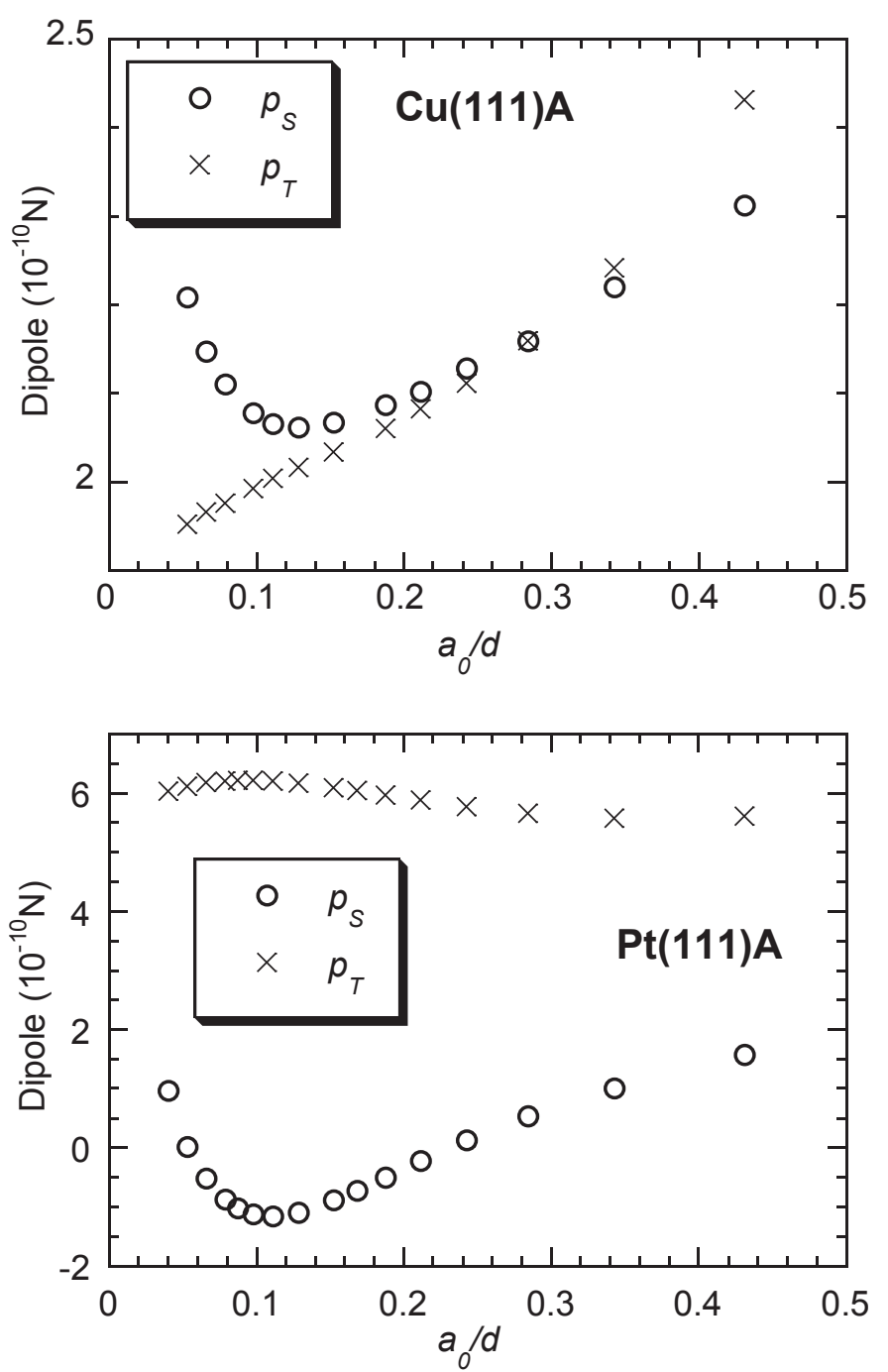

Figure 7. Variation with interstep distance of the value of the elastic dipoles that give the best fit of the atomic relaxations on $\mathrm{Cu}$ and $\mathrm{Pt}(111) \mathrm{A}$ vicinals. 


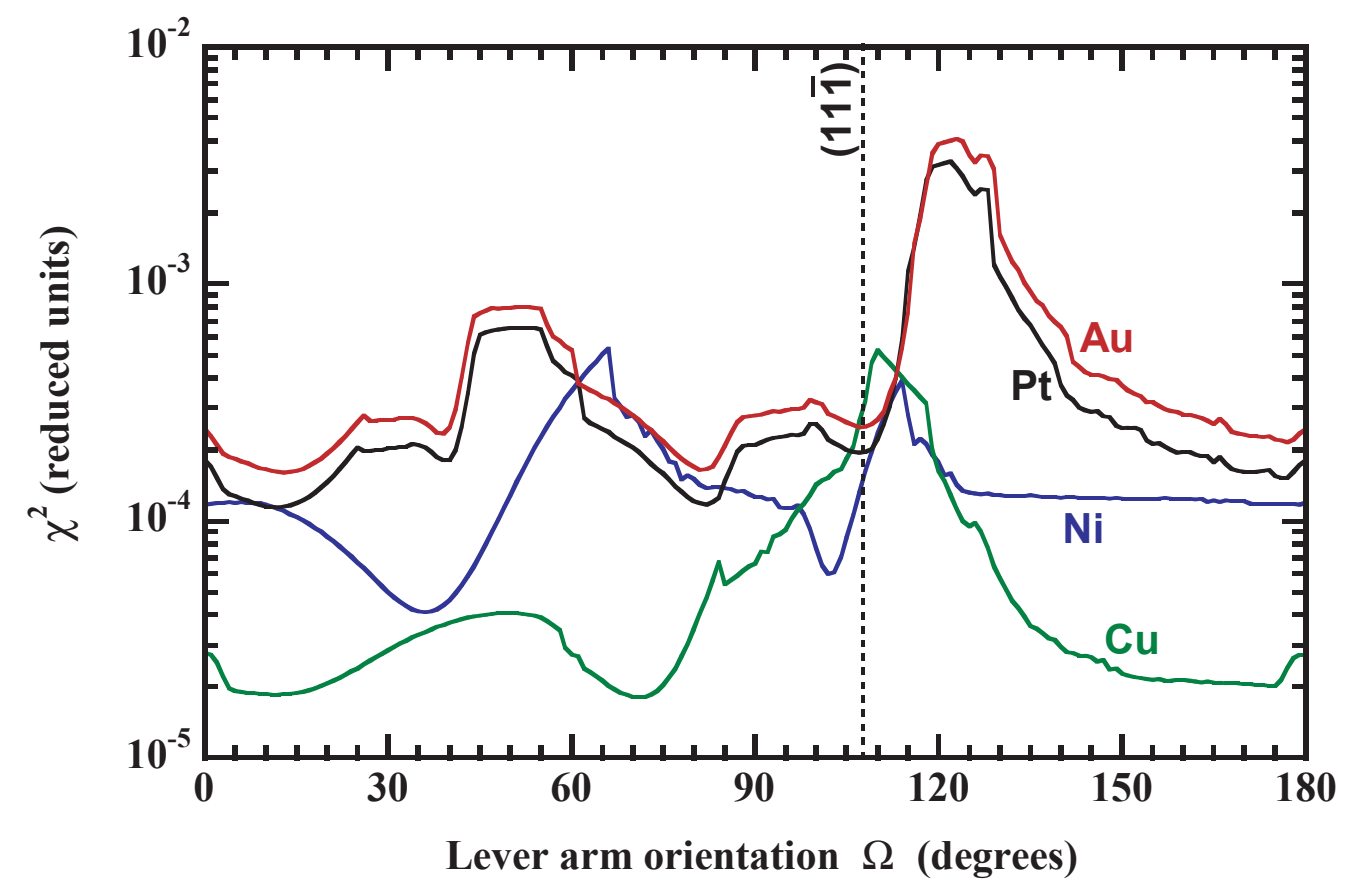

Figure 8 (color online). Variation of the $\chi^{2}$ for the fit of the atomic relaxations on $\mathrm{Cu}, \mathrm{Ni}, \mathrm{Pt}$ and $\mathrm{Au}(15,15,16)$ surfaces as function of the lever arm orientation with respect to the surface, $\Omega$. Blue: Ni; green: $\mathrm{Cu}$; black: Pt, red: Au. The step microfacet direction is indicated. 

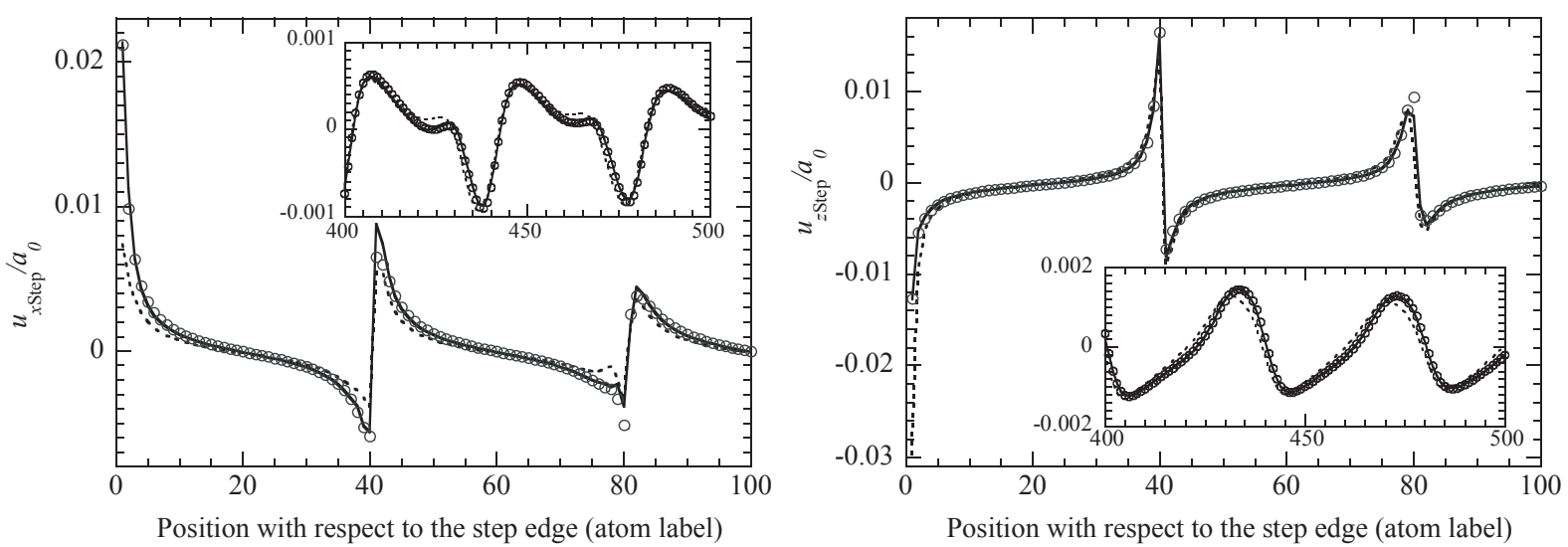

Figure 9. Comparison of the relaxation fields $\vec{u}_{\text {Step }}$ calculated by SMA and $\vec{u}_{\text {Dip }}(\vec{p})$ calculated by ALE for $\mathrm{Cu}(1179)$. Dots: $\vec{u}_{\text {Step }}$; dotted line: "geometrical buried dipole" model; continuous line: buried point dipole model. 


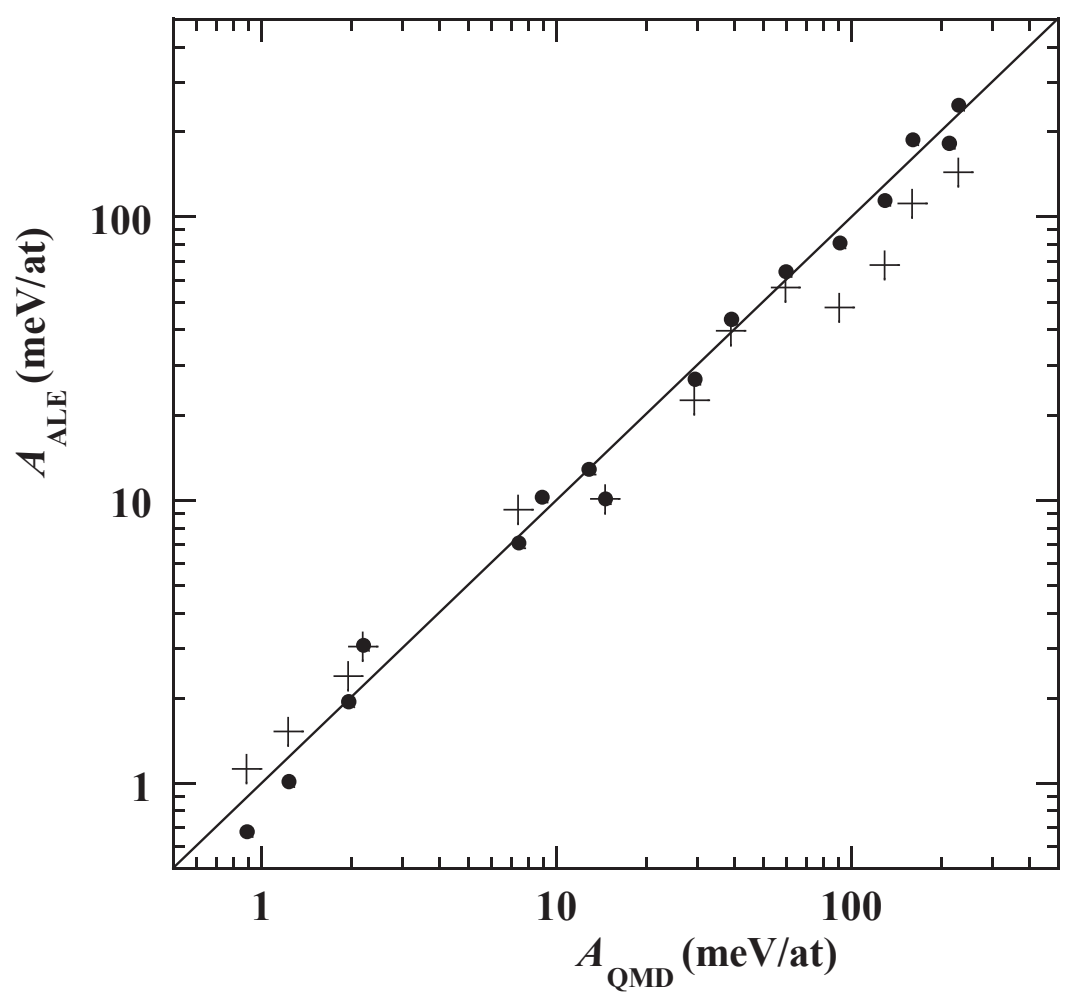

Figure 10. Comparison between the coefficients $A$ of the step interaction found by ALE for large interstep distances and by SMA from Eq. (6). Crosses: "geometrical buried dipoles"; dots: buried point dipoles. The values for the "geometrical buried dipoles" on Ni(111)A, $\mathrm{Ni}(111) \mathrm{B}$ and $\mathrm{Cu}(111) \mathrm{B}$ vicinals have not been given since a correct fit of the atomic displacements was not possible. 


\section{References}

${ }^{1}$ P. Gambardella, Ž. Šljivančanin, B. Hammer, M. Blanc, K. Kuhnke and K. Kern, Phys. Rev. Lett. 87, 056103 (2001).

${ }^{2}$ M. E. Fisher and D. S. Fisher, Phys. Rev. B 25, 3192 (1982).

${ }^{3}$ S. Papadia, M. C. Desjonquères, and D. Spanjaard, Phys. Rev. B 53, 4083 (1996).

${ }^{4}$ F. Raouafi, C. Barreteau, M. C. Desjonquères, and D. Spanjaard, Surf. Sci. 505, 183 (2002).

${ }^{5}$ R. Schmoluchowski, Phys. Rev. 60, 661 (1941).

${ }^{6}$ L. Peralta, E. Margot, Y. Berthier, and J. Oudar, J. Microsc. Spectrosc. Electron. 3, 151 (1978).

${ }^{7}$ C. Barreteau, F. Raouafi, M. C. Desjonquères and D Spanjaard, J. Phys.: Condens. Matter 15 (2003) S3171.

${ }^{8}$ V.I. Marchenko and A. Ya Parchin, Sov. Phys. JETP 52, 129 (1980).

${ }^{9}$ H.-C. Jeong, E. D. Williams, Surf. Sci. Rep. 34, 171 (1999).

${ }^{10}$ G. Prévot, P. Steadman and S. Ferrer, Phys. Rev. B 67, 245409 (2003).

${ }^{11}$ G. Prévot, A. Coati and Y. Garreau, Phys. Rev. B 70, 205406 (2004).

${ }^{12}$ G. Prévot and B. Croset, Phys. Rev. Lett. 92, 256104 (2004).

${ }^{13}$ V. Rosato, M. Guillopé, and B. Legrand, Phil. Mag. A 59, 321 (1989).

${ }^{14}$ G. Prévot, C. Cohen, D. Schmaus, P. Hecquet and B. Salanon, Surf. Sci. 506, 272 (2002).

${ }^{15}$ F. Cleri and V. Rosato, Phys. Rev. B 48, 22 (1993).

${ }^{16}$ P. Hecquet, Surf. Sci. 561, 127 (2004).

${ }^{17}$ S. Olivier, A. Saúl and G. Tréglia, Applied Surface Science 213, 866 (2003).

${ }^{18}$ S. Papadia, M. C. Desjonquères and D. Spanjaard, Phys Rev. B 53, 4083 (1996).

${ }^{19}$ C. Steimer, M. Giesen and H. Ibach, Surf. Sci. 471 (2001) 80.

${ }^{20}$ D.J. Bacon, D. M. Barnett, R. O. Scattergood, Prog. Mat. Sci. 23, 51 (1979).

${ }^{21}$ L. Landau and E. Lifchitz, Theory of Elasticity (Pergamon, Oxford 1970)

${ }^{22}$ B. Croset and G. Prévot, Phys. Rev. B 73, 045434 (2006). 